\title{
Static Stability Analysis Based on Probabilistic Power Flow Calculation considering P2G Technology
}

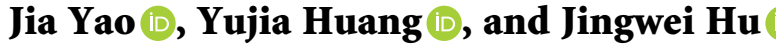 \\ College of Information Science and Engineering, Northeastern University, Shenyang, Liaoning 110819, China \\ Correspondence should be addressed to Jia Yao; 1970543@stu.neu.edu.cn
}

Received 17 January 2021; Revised 26 January 2021; Accepted 2 March 2021; Published 11 March 2021

Academic Editor: Dan Selisteanu

Copyright $\odot 2021$ Jia Yao et al. This is an open access article distributed under the Creative Commons Attribution License, which permits unrestricted use, distribution, and reproduction in any medium, provided the original work is properly cited.

\begin{abstract}
At present, integrated energy systems have received extensive attention, but there is no basic framework for stability analysis of coupled systems. The injection of a large amount of renewable energy also has a great impact on the stability of the system. This paper focuses on how to analyze the static stability of the coupling system with uncertainty, which mainly considers the uncertainty of wind power generation and photovoltaic power generation and also considers the influence of P2G technology on the whole system. Firstly, this paper analyzes the principles of wind power generation and photovoltaic power generation and constructs the probability model of renewable energy power generation power. Then, the three-point estimation method is used to process the data, and the probability distribution of the unknown quantity is obtained by probabilistic power flow analysis. Finally, the probability distribution of each eigenvalue is obtained by analyzing the sensitivity of the characteristic roots to the voltage. Thus, the static stability of the system is judged. The applicability of proposed methodology is demonstrated by analyzing an integrated IEEE 14-bus power system and a Belgian 20-node gas system in this paper.
\end{abstract}

\section{Introduction}

With the in-depth study of renewable energy [1, 2], wind power generation and photovoltaic power generation are connected to the grid in large numbers. But, the intermittent and random nature of renewable energy will have a great impact on the power grid.

The peak period of renewable energy is probably the low valley period of power grid load resulting in great waste. Considering that the reaction is unlikely to be complete, P2G technology will eventually produce a gas mixture of hydrogen and methane [3-6]. At present, many studies show that existing natural gas pipelines can be used to accept mixed gases generated by P2G technology [7]. Moreover, the mixed gas can be fed into the gas network to realize the bidirectional conversion between gas and electricity, which increases the coupling between the gas network and the power network. In this way, cross-regional and large-scale interconnection between electricity and gas can be realized, and the advantages of optimal energy allocation can also be realized $[8,9]$.

And, randomness is the uncertainty of exponential value. The generation power of renewable energy depends on the amount of energy at that time, and the amount of renewable energy is affected by many natural factors and is not controllable, which brings uncertain data to the system. This makes it difficult to judge the state of the system. It is very important whether the system can run stably $[10,11]$. The common way to obtain the running state of the system is the traditional power flow calculation, but the traditional power flow can not deal with the uncertain data brought by renewable energy, so it is necessary to use the uncertain power flow calculation to obtain the running state of the system.

At present, there are three methods for calculating probabilistic power flow: fuzzy mathematics method $[12,13]$, probabilistic power flow method [14], and interval analysis power flow method. The basic assumption of fuzzy distribution power flow is that the power demand boundary of the system bus is uncertain and obeys a certain probability distribution. Its solution set is some set of uncertain boundaries. The basic assumption of probabilistic power flow and interval power flow is that the power demand of the system bus is uncertain in a given interval. Among them, the basic mathematical theory of probabilistic power flow is probability theory. It represents uncertain data as a 
probability distribution and calculates its mean and variance. The uncertainty of known quantities is dealt by the Monte Carlo simulation method, semi-invariant method, point estimation method, and other data processing methods. Then, the probability distribution of the unknown quantity can be calculated by the power flow. It takes into account the random factors in system operation and can better represent the characteristics of power system operation than traditional power flow calculation, so as to facilitate us to find out the potential dangers and weak links of system operation.

Many papers have studied the probabilistic power flow calculation of wind power generation and photovoltaic power generation. In [15], the definition of probabilistic power flow calculation is proposed, and the most basic solution method is given. In [16], using the knowledge of probability theory, the probability distribution of the known quantity is analyzed, and the probability distribution of the unsolved quantity is finally obtained. The Markov chain quasi-Monte Carlo sampling method is adopted, and the correlation of power generation efficiency of multiple wind farms is considered to obtain the final probabilistic optimal power flow, in [17]. In consideration of the incomplete acceptance of wind power generation, in [18], a probabilistic power flow calculation method is propose based on the principle of proportional distribution and Latin hypercube sampling, which ensures the calculation accuracy and greatly improves the calculation speed.

The state of the system is often obtained by power flow calculation to judge the static stability of the system. Static stability refers to the ability of a system to operate stably after a small disturbance, which can be used to find the weak points of the system. In order to ensure the safe and reliable operation of the system, it is necessary to analyze the static stability of the system [19-21]. The randomness and intermittency of renewable energy will bring great fluctuation to the system and affect the static stability of the system. However, probabilistic power flow calculation is different from traditional power flow calculation. The result of uncertain power flow calculation is the probability distribution of system running state, not a set of fixed running values. So, this causes some difficulties to the static stability analysis.

Not only the power grid has static stability but also the gas network has static stability. Because the gas transported in the gas network is flammable and explosive, the stability of the gas network is also very important. But, P2G technology will use extra power to electrolyze water to get combustible gas and put the resulting mixture into the gas network. It will lead to changes in gas characteristics specific gravity (SG) and gross calorific value (GCV) and influence the operation of the gas grid. If a more accurate stability analysis of the integrated power-gas System is required, we should further the model of the gas network with gas mixture and calculate the power flow according to the new model. There are several papers on integrated power-gas systems. Yang et al. [22] modeled integrated power-gas systems, taking into account the uncertainty of pipeline parameters. Considering the different response time of gas network and power network, the optimal operation strategy is studied by power flow calculation, in [23].
To the best of authors' knowledge, there is little consideration that the stability of a system with uncertain data, and no consideration that the uncertainty of renewable energy and the consumption of renewable energy by $\mathrm{P} 2 \mathrm{G}$ technology's together. Of course, there also is no consideration that the static stability of integrated power-gas systems static with wind power generation and photovoltaic power generation. To solve these problems, the main contributions of this paper can be concluded as follows:

(1) An probabilistic power flow calculation method considering P2G technology is proposed. It not only considers the impact of the uncertainty of renewable energy on the system but also considers the impact of the gas mixture produced by P2G on the gas network. This makes the static stability analysis of the system more practical and more accurate.

(2) Based on probabilistic power flow calculation, the probability distribution of the power system static stability probability distribution of power system is analyzed. Considering the influence of uncertain data on power system, the confidence level of system stability can be obtained by analyzing the probability distribution of the unknown quantity. The potential dangers and weak links of power system operation can also be judged through the data.

(3) Not only the stability of electric power network but also the stability of gas network is considered. The uncertainty of renewable energy is analyzed, and its influence on the stability of gas network is obtained. In this way, it can effectively judge whether there is pipe blocking phenomenon in the gas network, so as to prevent accidents to some extent.

This paper is described in detail as follows. Treatment of uncertain data on wind and photovoltaic power generation is described in Section 2. An uncertain power flow calculation method considering P2G technology is described in Section 3. Static stability analysis based on uncertain power flow calculation is described in Section 4. Finally, the case studies are carried out on the integrated IEEE 14-bus power system and a Belgian 20-node gas system in Section 5, and conclusions are given in Section 6.

\section{Data Processing}

2.1. Wind Power Generation. The height of the weather station wind meter may not be the same as the height of the fan wheel shaft, so it is necessary to convert the wind speed measured by the wind meter into the wind speed at the fan wheel shaft height when calculating the wind power generated. The relationship between them can be expressed as

$$
v=v_{0}\left(\frac{h}{h_{0}}\right)^{a},
$$

where $v$ is the wind speed at fan shaft height, $h$ is the height of fan shaft, $v_{0}$ is the wind speed as measured by a wind gauge, $h_{0}$ is the height of the wind gauge, and $a$ is the roughness of the ground, which is usually $1 / 7$ in the open 
space. Some studies have shown that the average hourly wind speed in a year conforms to the Weibull distribution [24], but the study, which looked at 24 hours a day, suggests that turbulence, which varies in wind speed over short periods of time, cannot be ignored [25]. The intensity of turbulence can be expressed as

$$
I=\frac{\sigma_{v}}{\mu_{v}}
$$

where $\mu_{v}$ is the mean of wind speed and $\sigma_{v}$ is the standard deviation of the wind speed. Considering the influence of turbulence on wind speed, wind speed distribution can be expressed as a normal distribution. So, the wind speed probability density can be expressed as

$$
f(v)=\frac{1}{\sqrt{2 \pi} \sigma_{v}} e^{-\left(v-\mu_{v}\right)^{2} / 2 \sigma_{v}^{2}} .
$$

The relationship between the output power of wind power generation and wind speed can be expressed as

$$
P_{w}= \begin{cases}0, & v<v_{\text {in }}, \\ \frac{v-v_{\text {in }}}{v_{N}-v_{\text {in }}} P_{N}, & v_{\text {in }} \leq v \leq v_{N}, \\ P_{N}, & v_{N} \leq v \leq \text { out }, \\ 0, & v>v_{\text {out }},\end{cases}
$$

where $P_{N}$ is the rated power of wind turbines, $v_{\text {in }}, v_{N}$, and $v_{\text {out }}$ are cut in wind speed, rated wind speed, and cut out wind speed, respectively. The mean and the standard deviation of the output of the wind power generation can be expressed as

$$
\begin{aligned}
\mu_{\mathrm{pw}} & =\int_{0}^{+\infty} P_{w}(v) f(v) \mathrm{d} v, \\
\sigma_{\mathrm{pw}} & =\sqrt{\int_{0}^{+\infty}\left(P_{w}(v)-\mu_{\mathrm{pw}}\right)^{2} f(v) \mathrm{d} v .}
\end{aligned}
$$

2.2. Photovoltaic Power Generation. Daily exposure hours can be obtained from the data from weather stations. The total amount of daylight radiation throughout the day can be expressed as

$$
\begin{aligned}
H & =H_{L}\left(a+b \frac{s}{s_{L}}\right), \\
S_{L} & =\frac{2}{15} W_{s}, \\
H_{L} & =\tau H_{0},
\end{aligned}
$$

where $H_{L}$ is the intensity of solar radiation entering the Earth's atmosphere, a and $b$ are constant coefficients, $S_{L}$ is day length, $W_{s}$ is hour angle, $H_{0}$ is the radiation angle of the outer layer of the Earth's atmosphere, and $\tau$ is the air transparency coefficient. The air transparency coefficient generally fluctuates between 0.4 and 0.8 . Its probability density function can be expressed as

$$
f(\tau)=c \frac{\tau_{\max }-\tau}{\tau_{\max }} e^{\lambda \tau},
$$

where $\tau_{\max }$ is the maximum air transparency coefficient and $c$ and $\lambda$ are determined by the maximum air transparency coefficient and the mean value of air transparency coefficient. They can be represented as

$$
\begin{aligned}
& c=\frac{\lambda^{2} \tau_{\max }}{e^{\lambda \tau_{\max }}-\lambda \tau_{\max }-1}, \\
& \lambda=\frac{2 r-17.5119 e^{-1.3118 r}-1062 e^{-5.0426 r}}{\tau_{\max }}, \\
& r=\frac{\tau_{\max }}{\tau_{\max }-\mu_{\tau}},
\end{aligned}
$$

where $\mu_{\tau}$ is the mean of the air transparency coefficient. Therefore, if the total daily radiation amount is known, the probability density function of a day's light intensity can be expressed as

$$
R(t)=\frac{1}{\sqrt{2 \pi} \sigma_{R}} e^{-\left(\left(t-\mu_{R}\right)^{2} / 2 \sigma_{R}^{2}\right)} .
$$

Most of the existing studies use peak watt power to calculate the photovoltaic power generation. The daily exposure hours can be converted to peak watthours and expressed as

$$
D(t)=0.0116 H(t),
$$

where $D(t)$ is watts peak hours and 0.0116 is the conversion factor and in $\mathrm{h} \cdot \mathrm{cm}^{2} / \mathrm{cal}$. Therefore, the output power of the photovoltaic power can be expressed as

$$
P_{R}(t)=P_{f} \eta D(t)
$$

where $P_{f}$ and $\eta$ are the peak watt power and efficiency of the photovoltaic power generation respectively.

2.3. Three-Point Estimation Method. The three-point estimation method is the Taylor series expansion of the function $Y=h(X)$ associated with the $n$-dimensional random variable $X$. The probability density of $Y$ is obtained by estimating $Y$ at three points using the higher order moments of $X$. The taking positions and probabilities of these three points can be expressed as

$$
\left\{\begin{array}{l}
X_{i}=\left(\mu_{x}+\xi_{i} \cdot \sigma_{x}\right), \quad i=1,2,3, \\
P_{i}=\frac{(-1)^{3-i}}{\xi_{i}\left(\xi_{1}-\xi_{2}\right)}, \quad i=1,2, \\
P_{3}=1-P_{1}-P_{2},
\end{array}\right.
$$

where $\mu_{x}$ and $\sigma_{x}$ are the mean value and variance of $x$ variable, respectively, $P_{i}$ is the probability of taking the ith 
point position, and $\xi_{i}$ is the measurement coefficient of the position of the $i$ th point; it can be represented as

$$
\left\{\begin{array}{l}
\xi_{i}=\frac{\alpha_{3}}{2}+(-1)^{3-i} \sqrt{\alpha_{4}-\frac{3 \alpha_{3}^{2}}{4}}, \quad i=1,2, \\
\xi_{3}=0
\end{array}\right.
$$

where $\alpha_{i}$ can be expressed as

$$
\begin{aligned}
\alpha_{i} & =\frac{M_{i}(X)}{\sigma_{x}^{i}}, \\
M_{i}(X) & =\int_{-\infty}^{+\infty}\left(X-\mu_{x}^{i}\right) f(x) \mathrm{d} x,
\end{aligned}
$$

where $M_{i}(X)$ is the center distance and $f(x)$ is the probability density function. Because the three-point estimation method is used, the center distance and lambda coefficient are only required up to the fourth order. So, we know that $\alpha_{1}=0$ and $\alpha_{2}=1$ from the above formula. After determining the position of the point, the value of the unknown quantity at each point can be obtained by the function $Y=h(X)$. According to the probability of each point, the estimated values of the center distances of $Y$ can be expressed as

$$
E\left(Y^{j}\right) \approx \sum_{i=1}^{3} P_{i} \cdot[h(X)]^{i}
$$

So, the standard deviation of $Y$ can be expressed as

$$
\sigma_{Y}=\sqrt{E\left(Y^{2}\right)-E^{2}(Y)} .
$$

The uncertain data in the system are processed by the three-point estimation method, and the three pick-point positions of each uncertain node can be obtained. Then, substituting these three points into the power flow calculation, respectively, can obtain the estimated value of each point. Because one of the three pick points for each uncertain data is the mean, so if the number of the uncertain nodes in the system is $N$, the number of power flow calculations will be $2 \mathrm{~N}-1$.

\section{Probabilistic Power Flow Calculation}

3.1. Electric Network. In the electric network, in addition to the line parameter resistance and reactance, each node also corresponds to four variables, which are active power, reactive power, voltage amplitude, and voltage phase angle. According to Kirchhoff's law, the iterative equation of power flow calculation in electric network can be written as

$$
\left\{\begin{array}{l}
\Lambda P_{i}=P_{i}^{\mathrm{sp}}-P_{i}=P_{i}^{\mathrm{sp}}-V_{i} \sum_{j \in i} V_{j}\left(G_{i j} \cos \theta_{i j}+B_{i j} \sin \theta_{i j}\right), \\
\Lambda Q_{i}=Q_{i}^{\mathrm{sp}}-Q_{i}=Q_{i}^{\mathrm{sp}}-V_{i} \sum_{j \in i} V_{j}\left(G_{i j} \cos \theta_{i j}+B_{i j} \sin \theta_{i j}\right),
\end{array}\right.
$$

where $G$ is the electric conductance, $B$ is the electric inductance, $P$ is the active power, $Q$ is the reactive power, $V$ is the voltage amplitude, and $\theta$ is the voltage phase angle.

3.2. Gas Network. In the gas network, in addition to the line parameters, pipe length, pipe diameter, and friction coefficient, each node also corresponds to two variables, namely, air pressure and gas flow rate. The pressure drop function due to line parameters can be written as

$$
f_{i j}^{\mathrm{GL}}=C_{i j}^{\mathrm{GL}} \operatorname{sign}\left(\pi_{i}, \pi_{j}\right)\left(\operatorname{sign}\left(\pi_{i}, \pi_{j}\right) \cdot\left(\pi_{i}^{2}-\pi_{j}^{2}\right)\right)^{0.5},
$$

where $C_{i j}^{\mathrm{GL}}$ is the pipeline coefficient, $f$ is the gas-flow rate, $\pi$ is the gas pressure, and $\operatorname{sign}\left(\pi_{i}, \pi_{j}\right)$ is the sign function.

According to Kirchhoff's law, considering element characteristics and network topology constraints, the flow deviation equation of gas network nodes can be written as

$$
\Delta f_{i}=f_{i}^{\mathrm{GD}}+f_{i}^{\mathrm{GG}}+f_{i}^{\mathrm{CHP}}+f_{i}^{\mathrm{GB}}+f_{i}^{\mathrm{GS}}+\sum_{j=1}^{N_{\mathrm{GN}}} f_{i j}^{\mathrm{GL}}+\sum_{j=1}^{N_{\mathrm{GN}}} \tau_{i j}^{\mathrm{GC}},
$$

where $f_{i}^{\mathrm{GD}}$ is the gas flow from gas grid load consumption, $f_{i}^{\mathrm{GG}}$ is the gas flow from gas generator, $f_{i}^{\mathrm{CHP}}$ is the gas flow from gas turbines, $f_{i}^{\mathrm{GB}}$ is the gas flow from gas fired boiler, $f_{i}^{\mathrm{GS}}$ is the gas flow from gas source, $f_{i j}^{\mathrm{GL}}$ is the pipeline flow, and $\tau_{i j}^{\mathrm{GC}}$ is the flow rate consumed by gas compressors.

3.3. $22 G$. Because the gas mixture makes the $\mathrm{SG}$ and GCV in different positions of the gas network different, there is not only one variable of pressure at each gas node; but two variables of SG and GCV need to be increased. Therefore, the known quantities of relaxation nodes in the gas network are pressure, SG, and GCV, and the unknown quantities are gas flow rate, the known quantities of the load nodes in the gas network are the energy requirements, and the unknown quantities are the pressure, SG, and GCV. There will also be nonmixed air source and mixed air source in the gas network. The known quantities of nonmixed air sources are gas flow rate, SG, and GCV, and the unknown quantities are pressure. The known quantities of the mixed air source are the gas flow rate, and the unknown quantities are the pressure, SG, and GCV. 
According to the knowledge of fluid mechanics, SG and GCV of each node of gas network can also be obtained by iteration. This paper mainly considers the influence of the gas mixture of natural gas, hydrogen, and methane generated by $\mathrm{P} 2 \mathrm{G}$ on the gas network. So, SG and GCV can be expressed as

$$
\begin{aligned}
\Delta \mathrm{SG}_{i}= & \mathrm{SG}_{i}\left(f_{i}^{N}+f_{i}^{H}+f_{i}^{M}+\sum_{j=1}^{N_{0}} \mathrm{sg}\left(f_{j i}\right) \cdot f_{j i}\right) \\
& -\left(f_{i}^{N} \mathrm{SG}_{N}+f_{i}^{H} \mathrm{SG}_{H}+f_{i}^{M} \mathrm{SG}_{M}+\sum_{j=1}^{N_{0}} \mathrm{sg}\left(f_{j i}\right) \cdot\left(f_{j i} \mathrm{GCV}_{j}\right)\right)=0, \quad \forall i=1,2, \ldots,\left(N_{G}-1-N_{\text {non-mixed }}\right), \\
\Delta \mathrm{GCV}_{i}= & \mathrm{GCV}_{i}\left(f_{i}^{N}+f_{i}^{H}+f_{i}^{M}+\sum_{j=1}^{N_{0}} \mathrm{sg}\left(f_{j i}\right) \cdot f_{j i}\right) \\
& -\left(f_{i}^{H} \mathrm{GCV}_{N}+f_{i}^{H} \mathrm{GCV}_{H}+f_{i}^{M} \mathrm{GCV}_{M}+\sum_{j=1}^{N_{0}} \operatorname{sg}\left(f_{j i}\right) \cdot\left(f_{j i} \mathrm{GCV}_{j}\right)\right)=0, \quad \forall i=1,2, \ldots,\left(N_{G}-1-N_{\text {non-mixed }}\right),
\end{aligned}
$$

where SG and GCV, whose subscripts are $i$ and $j$, represents the value of the variable at node $i$ and node $j$, respectively, and $\operatorname{sg}\left(f_{j i}\right)$ is a sign function of gas flow where $\operatorname{sg}\left(f_{j i}\right)=1$ if $\operatorname{sg}\left(f_{j i}\right) \geq 0$ and 0 otherwise. $f_{i}^{N}, f_{i}^{H}$, and $f_{i}^{M}$ represent the gas flow of natural gas, hydrogen, and methane at node $i$, respectively. For more information, please refer to [3].

Therefore, the Jacobian matrix of power flow calculation for the gas mixture system can be expressed as

$$
J=\left(\begin{array}{cccccc}
\frac{\partial \Delta P}{\partial P} & \frac{\partial \Delta P}{\partial \theta} \frac{\partial \Delta P}{\partial V} & \frac{\partial \Delta P}{\partial \pi} & \frac{\partial \Delta P}{\partial \mathrm{SG}} & 0 \\
0 & \frac{\partial \Delta P}{\partial \theta} \frac{\partial \Delta P}{\partial V} & 0 & 0 & 0 \\
\frac{\partial \Delta f}{\partial P} & 0 & 0 & \frac{\partial \Delta f}{\partial \pi} & \frac{\partial \Delta f}{\partial \mathrm{SG}} & \frac{\partial \Delta f}{\partial \mathrm{GCV}} \\
\frac{\partial \Delta \mathrm{SG}}{\partial P} & 0 & 0 & \frac{\partial \Delta \mathrm{SG}}{\partial \pi} & \frac{\partial \Delta \mathrm{SG}}{\partial \mathrm{SG}} & 0 \\
\frac{\partial \Delta \mathrm{GCV}}{\partial P} & 0 & 0 & \frac{\partial \Delta \mathrm{GCV}}{\partial \pi} & 0 & \frac{\partial \Delta \mathrm{GCV}}{\partial \mathrm{GCV}}
\end{array}\right)
$$

\section{Static Stability Analysis}

4.1. Confidence Interval and Confidence Level. A confidence interval of a probability sample is an interval estimate of a population parameter of this sample. The confidence interval shows the degree to which the true value of this parameter has a certain probability of falling around the measurement result. The confidence interval gives the confidence of the measured value of the measured parameter, that is, the "certain probability". This probability is called the confidence level.
For any system, there is no unique state in which it can reliably operate. Instead, there will be a range within which the system can operate stably. The static stability analysis is to judge whether the running state of the system at that time is within this stable range. And, the uncertainty of data will cause the fluctuation of system running state, so it is impossible to directly judge whether the running state of the system can be in a stable range. In this case, whether the running state of the system is within this range becomes a probabilistic problem. To analyze the static stability of such uncertain system is to analyze the probability that its running state is within the stable range. These are confidence intervals and confidence levels in probabilistic knowledge. The confidence interval we choose is the range that the system can operate stably. The confidence level of system stability is the probability that the system operating state is within this confidence interval.

4.2. Electric Grid Stability Judgment. Existing studies have shown that the static stability of the power system can be judged by calculating the eigenvalue of the Jacobian matrix of power flow [26]. In this paper, the probability distribution of the characteristic roots of the system can be obtained by analyzing the results of the probabilistic power flow. Then, the confidence level of the stability of the whole system can be obtained by the stability criterion. First, the characteristic roots of the Jacobian matrix calculated by each power flow are obtained, and then the mean value of each eigenvalue is obtained according to the probability of taking points. It can be represented as

$$
\lambda_{k} \approx \sum_{i=1}^{3} P_{i} \cdot \lambda(x) .
$$

And, the covariance matrix of the characteristic roots can be expressed as 


$$
C_{\lambda}=J_{\lambda} C_{v} J_{\lambda}^{T}
$$

where $C_{\lambda}$ is the covariance matrix of the characteristic roots, $J_{\lambda}$ is the Jacobian matrix between the characteristic roots and the voltage, and $C_{v}$ is the covariance matrix of voltage. They can be represented as

$$
\begin{aligned}
{\left[\begin{array}{c}
\lambda_{1} \\
\vdots \\
\lambda_{n}
\end{array}\right] } & =J_{\lambda}\left[\begin{array}{c}
V_{1} \\
\vdots \\
V_{n}
\end{array}\right] C_{v a b}=\operatorname{cov}\left(V_{a}, V_{b}\right), \quad a, b=1,2, \ldots, n, \\
C_{v a b} & =\operatorname{cov}\left(V_{a}, V_{b}\right), \quad a, b=1,2 \ldots, n .
\end{aligned}
$$

The probability distribution of each node voltage can be obtained by the above formula, so as to determine how much probability the whole power network can run stably.

4.3. Gas Grid Stability Judgment. The gas transmission pressure of natural gas will affect the operating pressure of the pipeline itself. Appropriate operating pressure is an important guarantee to ensure the stable work and safety of the pipeline. When the gas transmission pressure of natural gas is too large or greater than the normal gas transmission pressure, the gas transmission pressure will have a great impact on the pipeline, increase the flow rate of the fluid, and may directly lead to the fall off or rupture of the pipeline interface. If the gas transmission pressure of the pipeline is too small and the pressure is not enough to reach the normal operating pressure, then the gas transmission pressure cannot make the pipeline carry out normal pipeline transportation.

Therefore, in the transportation of the gas network, the pressure value of each node of the gas network has the greatest influence on the safety. The influence of the fluctuation brought by renewable energy on the stability of the gas network can be judged by judging the probability distribution of pressure of each node in the gas network:

$$
\begin{aligned}
& E_{p} \approx \sum_{i=1}^{3} P_{i} \cdot p_{k}, \\
& \sigma_{p}=\sqrt{E\left(p^{2}\right)-E^{2}(p)},
\end{aligned}
$$

where $E_{p}$ and $\sigma_{p}$ are the mean value and variance of pressure.

\section{Numerical Simulations}

In this paper, the feasibility of this method is verified by an integrated IEEE 14-bus power system and a Belgian 20-node system, which is shown in Figure 1. For the IEEE 14-bus power system, it has two GfG units (GfG1 at E1 and GfG2 at E2), one wind turbine at E3 and one photovoltaic generator at E6 in this paper. For the Belgian 20-node gas system [27], the gas well at G1 provides traditional natural gas and serves as a slack node with the reference pressure of 56 bar. The gas well at G5 and G8 provides synthetic natural gas and its SG and GCV are known. And, the gas well at $G 2, G 13$, and $G 14$ provides the mixture of synthetic natural gas and hydrogen whose SG and GCV are unknown. It is assumed that there is no gas leakage and the gases are completely mixed without chemical reaction. The pipeline parameters and gas load demands are given in [28]. The SG and GCV of different gas supply source are shown in Table 1.

5.1. Renewable Energy Power Probability Density. The data used in this paper are from a group of wind power generation with 30 wind turbines. The rated power of each wind turbines is $2.5 \mathrm{MW}$. The cut in wind speed, rated wind speed, and cut out wind speed are, respectively, $13 \mathrm{~m} / \mathrm{s}, 7.3 \mathrm{~m} / \mathrm{s}$, and $13 \mathrm{~m} / \mathrm{s}$. The mean value of wind speed data is $5 \mathrm{~m} / \mathrm{s}$, and the standard deviation is $8 \mathrm{~m} / \mathrm{s}$. Thus, the probability density function of the output power of the wind generator can be obtained, which is shown in Figure 2.

In this paper, the photovoltaic generator is located at $104.4^{\circ} \mathrm{E}$ and $30.4^{\circ} \mathrm{N}$. The loss coefficient is 0.85 . The average temperature during the day is $24^{\circ} \mathrm{C}$. It is also possible to plot the probability density of the output power of the photovoltaic generator, which is shown in Figure 3.

5.2. Static Stability Analysis of Electric System. Using the three-point estimation method to process the data, we can obtain the three-point location and probability of wind power generation and photovoltaic power generation. And then, plugging those points into the power flow equation can get five sets of results. Finally, the probability distribution of eigenvalues calculated by each power flow can be analyzed. Because the whole power system has 14 nodes, including one slack node, four PV nodes, and nine PQ nodes, there are 22 power flow equations in the power system, namely, 22 characteristic roots. There are nine pairs of conjugate characteristic roots, the 1 st and $2 \mathrm{nd}$, the 4 th and 5 th, the 7 th and 8 th, the 10th and 11th, the 12th and 13 th, the 14 th and 15 th, the 16th and 17th, the 19th and 20 th, and the 21 st and $22 \mathrm{nd}$. Since the static stability of the system depends on the real part of each characteristic root, the effect of a pair of conjugate characteristic roots on the stability of the system is the same. The probability distribution of the characteristic root real part can be obtained by analyzing the five groups of characteristic root values, which is shown in Figure 4. According to the curve in the figure, it can be found that each characteristic root has a high probability of falling on the negative axis, so the power system has a good stability.

5.3. Static Stability Analysis of Gas System. The impact of renewable energy uncertainty on SG and GCV of each node can be analyzed through the data obtained from the above power flow calculation. Finally, the mean values of SG and GCV at each node is shown in Table 2. The fluctuation trend of SG of node 14 affected by uncertain information is shown in Figure 5, and the fluctuation trend of GCV is in Figure 6.

By analyzing the pressure value of each node in the gas network, the probability distribution can be obtained, which 


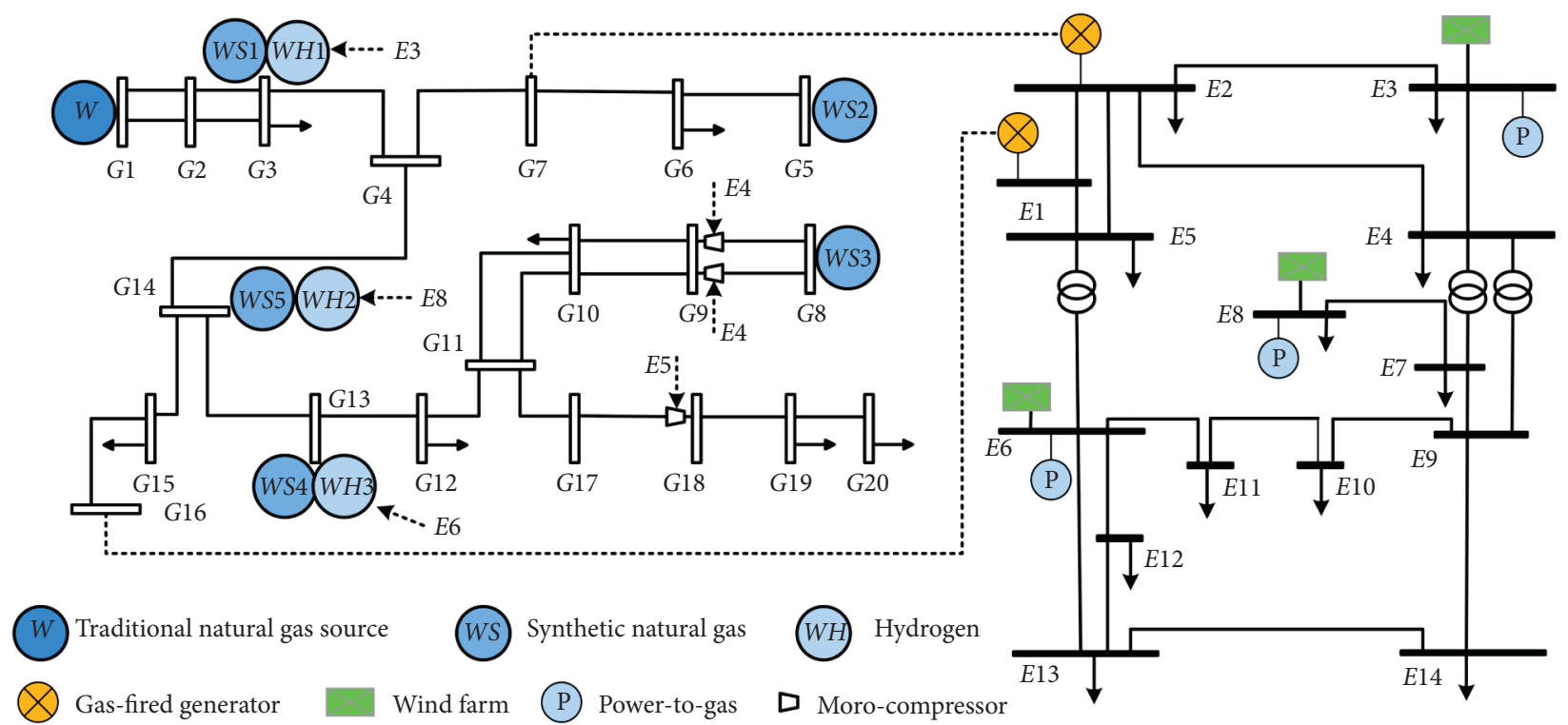

FIGURE 1: Integrated IEEE 14-bus system and Belgian 20-node gas system.

TABLE 1: SG and GCV of gas sources.

\begin{tabular}{lccc}
\hline & Natural gas & Synthetic natural gas & Hydrogen \\
\hline SG & 0.6048 & 0.58 & 0.0696 \\
GCV & 41.04 & 37.4 & 12.75 \\
\hline
\end{tabular}

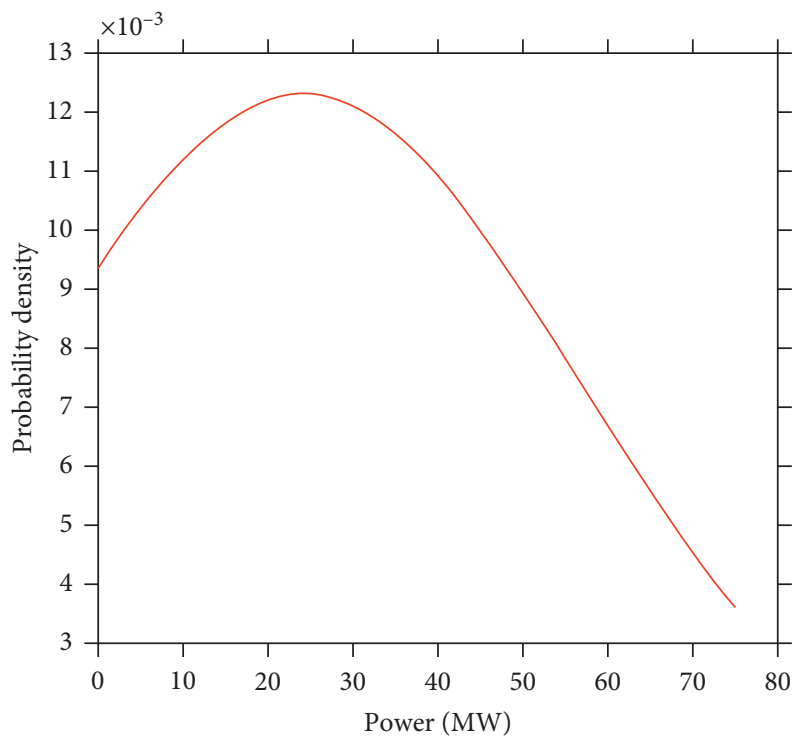

FIgURe 2: Probability density function of wind photovoltaic power generation.

is shown in Figure 7. It can be seen that the pressure of each node fluctuates in a certain range. The higher the ordinate, the greater the possibility that the pressure of the node falls there, the more stable the node is. It is obvious that the pressure of Node 3 is the most stable, and Figure 8 is the curve of Node 3.
Figure 9 compares Node 3, Node 19, and Node 20. It can be clearly seen that compared with Node 3 , node 19 , and Node 20 have great pressure changes. It can be seen that the stability of node 19 and node 20 is very poor, which is the weak link of the system. This shows that the nodes at the end of the network are very fragile. When we pay attention to the 


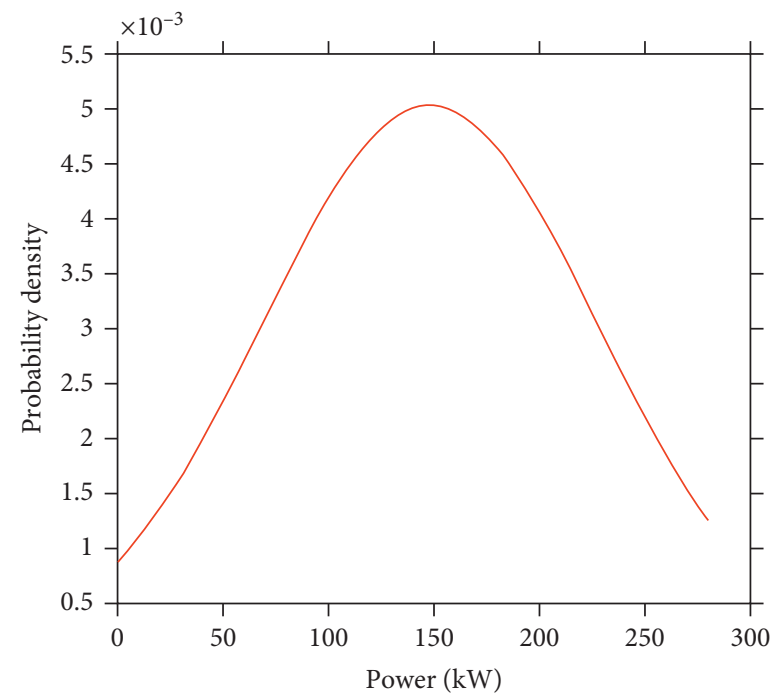

FIgure 3: Probability density function of photovoltaic power generation.

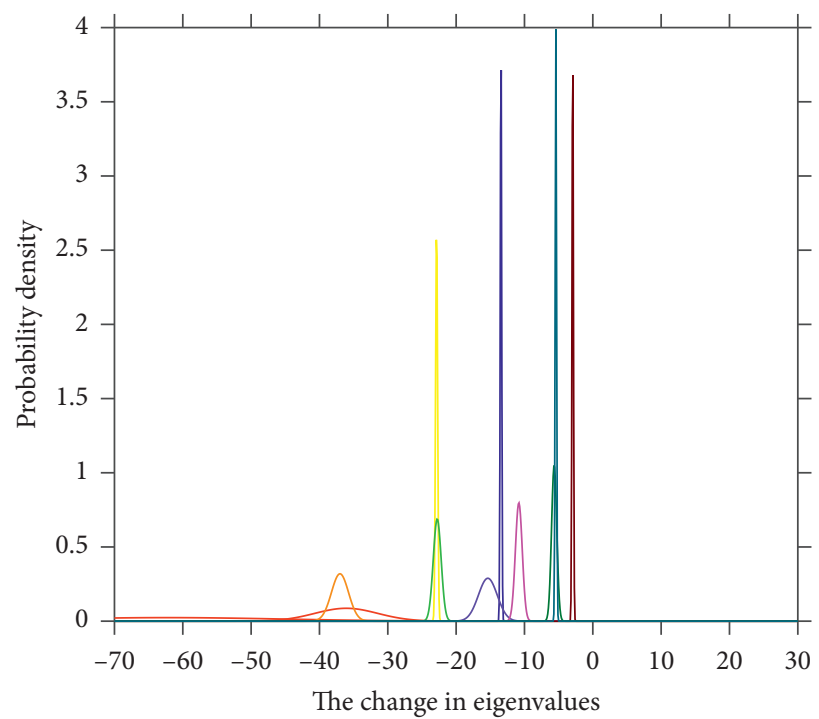

- Eigenvalues 1\&2

— Eigenvalues 3

— Eigenvalues $4 \& 5$ Eigenvalues 6

— Eigenvalues 7\&8

_ Eigenvalues 9

_ Eigenvalues 10\&11

FIGURe 4: Probability distribution of the eigenvalue.

TABLE 2: SG and GCV at different nodes.

\begin{tabular}{lccccc}
\hline Node & SG & GCV & Node & SG & GCV \\
\hline 1 & 0.6048 & 41.04 & 11 & 0.58 & 37.4 \\
2 & 0.5972 & 39.9413 & 12 & 0.58 & 37.4 \\
3 & 0.5972 & 39.9413 & 13 & 0.58 & 37.398 \\
4 & 0.5972 & 39.9413 & 14 & 0.5883 & 38.6338 \\
5 & 0.58 & 37.4 & 15 & 0.5883 & 38.6338 \\
6 & 0.5861 & 38.2952 & 16 & 0.5883 & 38.6338 \\
7 & 0.5972 & 39.9413 & 17 & 0.58 & 37.4 \\
8 & 0.58 & 37.4 & 18 & 0.58 & 37.4 \\
9 & 0.58 & 37.4 & 19 & 0.58 & 37.4 \\
10 & 0.58 & 37.4 & 20 & 0.58 & 37.4 \\
\hline
\end{tabular}

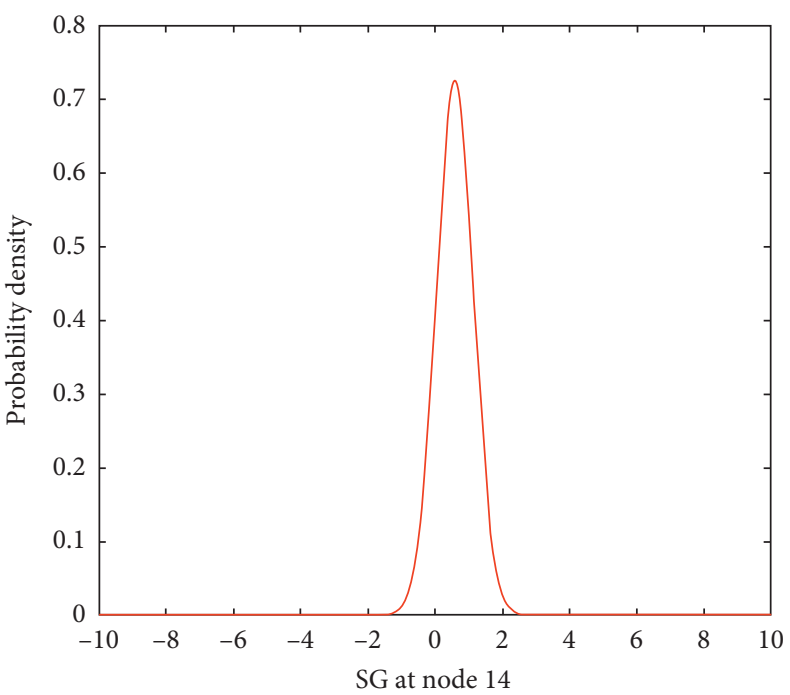

SG

Figure 5: SG at node 14.

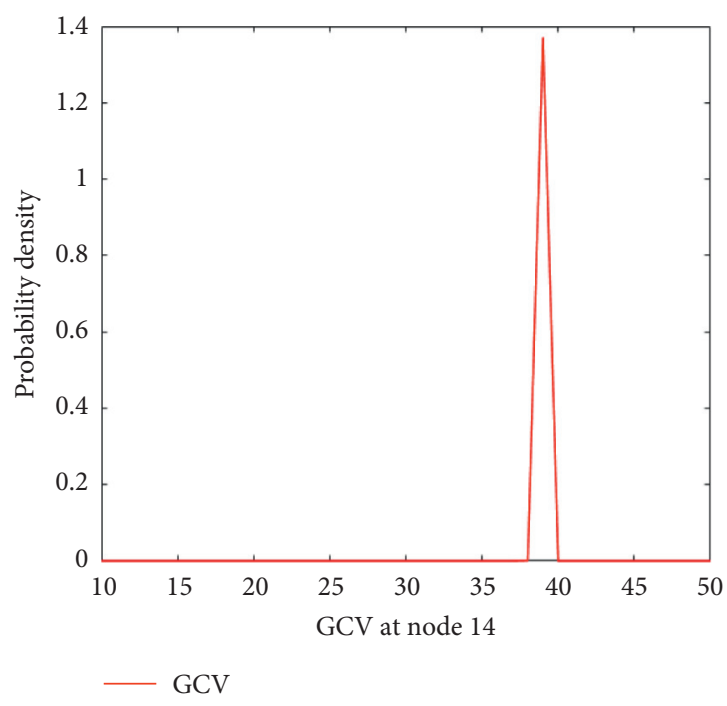

Figure 6: GCV at node 14. 


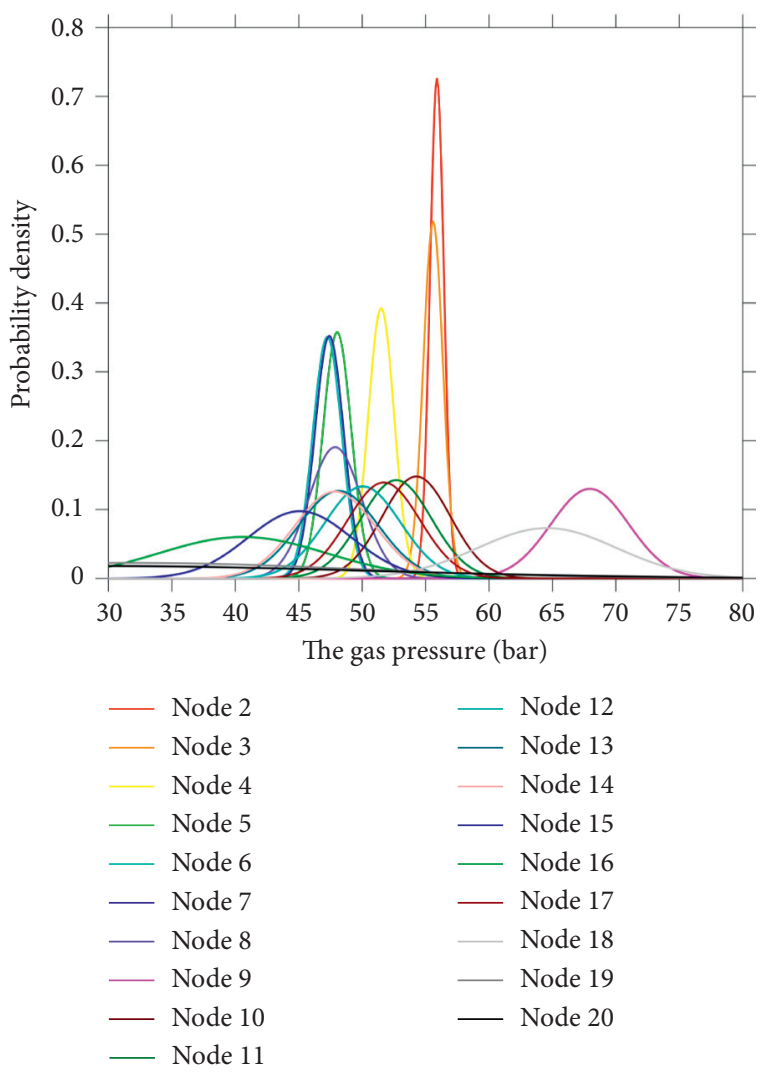

FIgURE 7: Probability distribution of the eigenvalue.

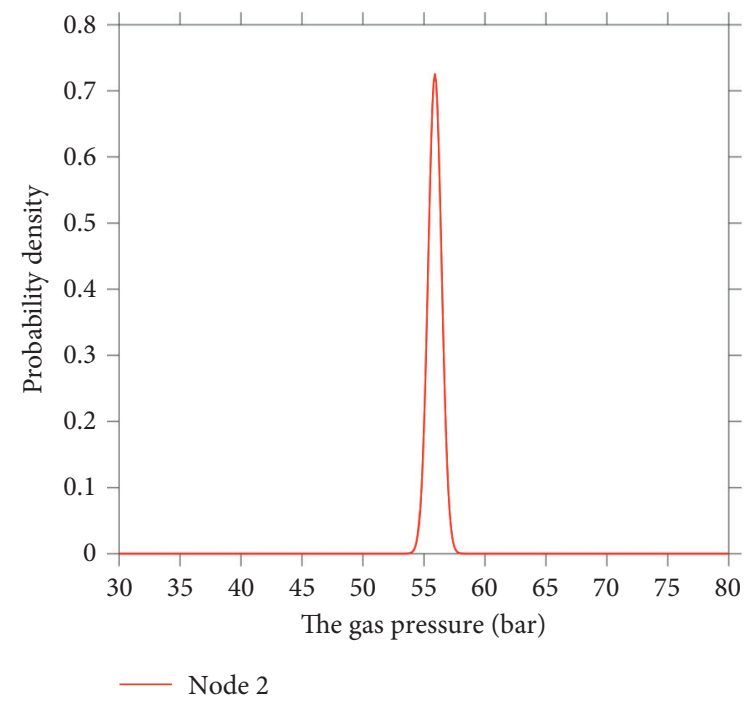

Figure 8: Probability distribution of pressure at node 3.

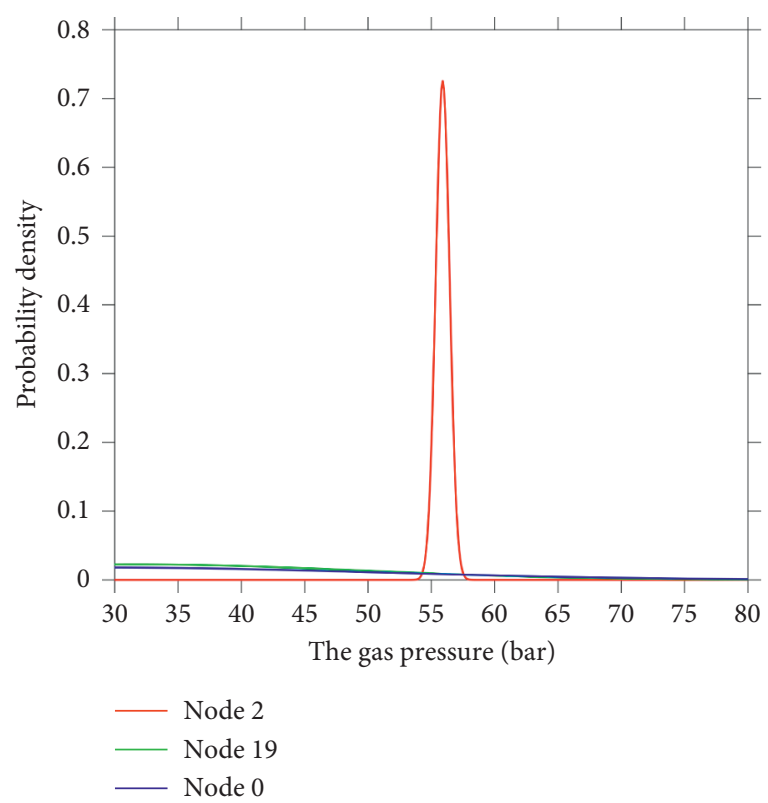

Figure 9: Comparison among Nodes 3, 19, and 20.

stability of the whole gas network, we should pay attention to the stability of the terminal nodes.

\section{Conclusion}

This paper constructed a coupling network model considering renewable energy generation and P2G technology and did a static stability analysis of this model. This model takes into account the influence of renewable power generation uncertainty on the system. It also takes into account the impact of P2G technology on the gas characteristics specific gravity and gross calorific value of the whole network. In this paper, the static stability of this model is analyzed by probabilistic power flow calculation and characteristic root sensitivity method. The influence of data uncertainty on the stability of power network and gas network is analyzed, respectively. The confidence level of the system can be obtained by determining the confidence interval of power network and gas network. Thus, the potential danger and weak link of the system can be further judged. The feasibility of this method is verified by simulation based on actual data. The effects of the randomness and intermittency of renewable energy on the stability of the system are visually demonstrated.

\section{Data Availability}

The data used to support the findings of this study are included within the article. 


\section{Conflicts of Interest}

The authors declare they have no conflicts of interest.

\section{Acknowledgments}

This work was supported by the National Key R\&D Program of China under grant (2018YFA0702200), the Key Projects of National Natural Science Foundation of China (U20A20190), the National Natural Science Foundation of China (62073065), and the Liaoning Province to Revitalize the Liao Talents Plan (XLYC1802046).

\section{References}

[1] Q. Sun and L. Yang, "From independence to interconnectionA review of AI technology applied in energy systems," CSEE Journal of Power and Energy Systems, vol. 5, no. 1, pp. 21-34, 2019.

[2] Q. Sun, N. Zhang, S. You, and J. Wang, "The dual control with consideration of security operation and economic efficiency for energy hub," Institute of Electrical and Electronics Engineers Transactions on Smart Grid, vol. 10, no. 6, pp. 59305941, 2019.

[3] Y. Huang, Q. Sun, N. Zhang, and R. Wang, "A multi-slack bus model for bi-directional energy flow analysis of integrated power-gas systems," CSEE Journal of Power and Energy Systems, to be published.

[4] N. Zhang, Q. Sun, J. Wang, and L. Yang, "Distributed adaptive dual control via consensus algorithm in the energy internet," Institute of Electrical and Electronics Engineers Transactions on Industrial Informatics, vol. 2020, 1 page, 2020.

[5] A. Sawas and H. E. Z. Farag, "Optimal sizing of powerctocgas units toward elevated renewable power penetration," in Proceedings of the 2019 Institute of Electrical and Electronics Engineers Canadian Conference of Electrical and Computer Engineering (CCECE), pp. 1-4, Edmonton, CA, USA, May 2019.

[6] Z. Zeng, T. Ding, Y. Xu, Y. Yang, and Z. Dong, "Reliability evaluation for integrated power-gas systems with power-togas and gas storages," Institute of Electrical and Electronics Engineers Transactions on Power Systems, vol. 35, no. 1, pp. 571-583, 2020.

[7] T. A. Williams, Interchangeability of Fuel Gases, New YorkThe Industrial Press, New York, NY, USA, 1965.

[8] G. Guandalini, S. Campanari, and M. C. Romano, "Power-to-gas plants and gas turbines for improved wind energy dispatchability: energy and economic assessment," Applied Energy Barking Then Oxford, vol. 147, pp. 117-130, 2015.

[9] J. Liu, H. Zhong, K. Zeng, H. Fan, and Q. Chen, “Optimal scheduling of multiple energy system considering power to gas unit," in Proceedings of the 2017 IEEE Conference on Energy Internet and Energy System Integration (EI2), pp. 1-6, Beijing, China, November 2017.

[10] R. Wang, Q. Sun, W. Hu, Y. Li, D. Ma, and P. Wang, "SoCbased droop coefficients stability region analysis of the battery for stand-alone supply systems with constant power loads," Institute of Electrical and Electronics Engineers Transactions on Power Electronics, vol. 2020, 1 page, 2020.

[11] R. Wang, Q. Sun, D. Ma, and Z. Liu, "The small-signal stability analysis of the droop-controlled converter in electromagnetic timescale," Institute of Electrical and Electronics Engineers
Transactions on Sustainable Energy, vol. 10, no. 3, pp. 14591469, 2019.

[12] X. Guan, W.-H. E. Liu, and A. D. Papalexopoulo, "Application of a fuzzy set method in an optimal power flow," Electric Power Systems Research, vol. 34, no. 1, pp. 11-18, 1995.

[13] G. K. V. Raju and P. R. Bijwe, "Fuzzy distribution power flow for weakly meshed systems," in Proceedings of the 2007 IEEE Power Engineering Society General Meeting, p. 1, Tampa, FL, USA, June 2007.

[14] J. M. Morales, L. Baringo, A. J. Conejo, and R. Minguez, "Probabilistic power flow with correlated wind sources flow with correlated wind sources," IET Generation, Transmission \& Distribution, vol. 4, no. 5, pp. 641-651, 2010.

[15] A. P. S. Meliopoulos, G. J. Cokkinides, and X. Y. Chao, "A new probabilistic power flow analysis method flow analysis method," Institute of Electrical and Electronics Engineers Transactions on Power Systems, vol. 5, no. 1, pp. 182-190, 1990.

[16] K. Qian, C. Zhou, M. Allan, and Y. Yuan, "Modeling of load demand due to ev battery charging in distribution systems," Institute of Electrical and Electronics Engineers Transactions on Power Systems, vol. 26, no. 2, pp. 802-810, 2011.

[17] Z. Ren, W. Yan, C. Ding, J. Yu, and X. Zhao, "Probabilistic optimal power flow analysis of virtual power plant containing photovoltaic generation," in Proceedings of the 2014 IEEE PES Asia-Pacific Power and Energy Engineering Conference (APPEEC), pp. 1-5, Kowloon, Hong Kong, December 2014.

[18] J. Yu, H. Qi, S. Qiu, X. Wang, and H. Zhang, "Probabilistic load flow calculation with irregular distribution variables considering power grid receivability of wind power generation," in Proceedings of the 2016 IEEE 8th International Power Electronics and Motion Control Conference (IPEMC-ECCE Asia), pp. 1457-1461, Hefei, China, May 2016.

[19] Y. Huang and Q. Sun, "Static voltage stability analysis for integrated energy system," Chinese Journal of Electrical Engineering, vol. 39, no. 1, pp. 44-53, 2019.

[20] R. Wang, Q. Sun, W. Hu, Y. Li, D. Ma, and P. Wang, "SoCbased droop coefficients stability region analysis of the battery for stand-alone supply systems with constant power loads," Institute of Electrical and Electronics Engineers Transactions on Power Electronics, vol. 2021, 1 page, 2021.

[21] W. Hu, C. Ruan, H. Nian, and D. Sun, "Zero-sequence current suppression strategy with common mode voltage control for open-end winding PMSM drives with common DC bus," Institute of Electrical and Electronics Engineers Transactions on Industrial Electronics, vol. 2020, 1 page, 2020.

[22] L. Yang, X. Zhao, X. Hu, G. Sun, and W. Yan, "Probabilistic power and gas flow analysis for electricity-gas coupled networks considering uncertainties in pipeline parameters," in Proceedings of the 2017 IEEE Conference on Energy Internet and Energy System Integration (EI2), pp. 1-6, Beijing, China, November 2017.

[23] J. Fang, "Dynamic optimal energy flow in the integrated natural gas and electrical power systems," in Proceedings of the 2018 IEEE Power Energy Society General Meeting (PESGM), p. 1, Atlanta, GA, USA, August 2018.

[24] W. E. Dickinson, Solar Energy Technology Handbook, Butterworth, London, UK, 1980.

[25] T. Burton, N. Jenkins, D. Sharpe, and E. Bossanyi, Wind Energy Handbook, John Wiley and Sons Ltd, Chichester UK, 2001.

[26] A. Shabanpour-Haghighi and A. R. Seifi, “An integrated steady-state operation assessment of electrical, natural gas, and district heating networks," Institute of Electrical and 
Electronics Engineers Transactions on Power Systems, vol. 31, no. 5, pp. 3636-3647, 2016.

[27] C. M. Correa-Posada and P. Snchez-Martn, "Integrated power and natural gas model for energy adequacy in short-term operation," Institute of Electrical and Electronics Engineers Transactions on Power Systems, vol. 30, no. 6, pp. 3347-3355, 2014.

[28] D. De Wolf and Y. Smeers, "The gas transmission problem solved by an extension of the simplex algorithm," Management Science, vol. 46, no. 11, pp. 1454-1465, 2000. 